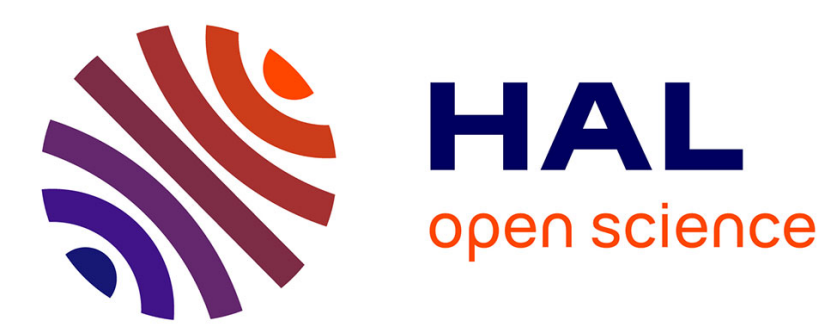

\title{
Frequency comb customization by controlling the optical injection dynamics
}

Yaya Doumbia, Tushar Malica, Delphine Wolfersberger, Krassimir Panajotov, Marc Sciamanna

\section{- To cite this version:}

Yaya Doumbia, Tushar Malica, Delphine Wolfersberger, Krassimir Panajotov, Marc Sciamanna. Frequency comb customization by controlling the optical injection dynamics. SPIE Photonics Europe, Apr 2020, Strasbourg, France. hal-03132189

\section{HAL Id: hal-03132189}

https://hal-centralesupelec.archives-ouvertes.fr/hal-03132189

Submitted on 4 Feb 2021

HAL is a multi-disciplinary open access archive for the deposit and dissemination of scientific research documents, whether they are published or not. The documents may come from teaching and research institutions in France or abroad, or from public or private research centers.
L'archive ouverte pluridisciplinaire HAL, est destinée au dépôt et à la diffusion de documents scientifiques de niveau recherche, publiés ou non, émanant des établissements d'enseignement et de recherche français ou étrangers, des laboratoires publics ou privés. 


\title{
Frequency comb customization by controlling the optical injection dynamics
}

\author{
Yaya Doumbia $^{\text {a, b }}$, Tushar Malica ${ }^{\text {a, b }}$, Delphine Wolfersberger ${ }^{\text {a, b }}$, Krassimir Panajotov ${ }^{\text {, d }}$, and \\ Marc Sciamanna ${ }^{a}$, b \\ ${ }^{a}$ Chaire Photonique, LMOPS, CentraleSupélec, Metz, France \\ ${ }^{\mathrm{b}}$ Université de Lorraine, CentraleSupélec, LMOPS, Metz, France \\ ${ }^{c}$ Brussels Photonics Group (B-PHOT), Vrije Universiteit Brussel, Brussels, Belgium \\ ${ }^{\mathrm{d}}$ Institute of Solid State Physics, Bulgarian Academy of Sciences, Sofia, Bulgaria
}

\begin{abstract}
This work reports on numerical analysis of the nonlinear dynamics of a semiconductor laser subject to an optical injection with a frequency comb. We identified the fundamental difference between the single mode injection and frequency comb injection. By varying the injection parameters (detuning and injection ratio), the results show that the slave laser is able to reproduce the injected frequency comb (new comb) in different parameters regions. The appearance of the new comb depends on the number of injected comb lines for fixed comb properties.
\end{abstract}

Keywords: Optical injection locking, Frequency comb, Times periodic dynamics, choas.

\section{INTRODUCTION}

Optical injection has been used to study the different dynamical outputs of a semiconductor laser for many years. Firstly, as a way to improve the coherence properties of a diode laser, but more recently as a technique to explore the specific dynamics such as optical injection locking, ${ }^{1}$ bifurcation scenarios ${ }^{2,3}$ and optical chaos. ${ }^{1,4}$ These nonlinear dynamics lead to applications in several fields such as cryptography based on the optical chaos, ${ }^{5}$ optical communication ${ }^{6}$ and generation of optical frequency comb. ${ }^{7}$ It is known that the optical injection with a single mode laser in the multimode lasers provides additional dynamics including polarisation dynamics of VCSELs $^{8-10}$ and longitudinal mode dynamics in quantum dot lasers. ${ }^{11,12}$ By contrast, there are very few studies about the dynamics of a single mode laser being injected with a multimode laser. The stability of the frequency comb due to their fixed comb spacing and constant relation phase make them a perfect candidate for such configuration.

The optical injection locking has been proposed as a technique of demultiplexing of an optical frequency comb by injecting it in the single mode laser cavity. ${ }^{13,14}$ The optical injection with a frequency comb leads to the selective amplification of the comb line that shows the smallest detuning from the slave laser. Recent experimental and theoretical work has focused on the effects of the injection parameters (injection ratio and detuning frequency) and the comb spacing on the side mode suppression ratio. ${ }^{15,16}$ A numerical investigation on the nonlinear dynamics of a single mode laser subjected to a frequency comb injection reveals the existence of a time periodic dynamics which depends of the injection parameters and properties of the comb. ${ }^{17}$

In this paper we present a numerical analysis of the specific dynamics of a single mode laser with frequency comb injection. Firstly, we explore the fundamental differences between injection locking solution in the case of a single mode laser as slave laser subjected to a single mode injection and to a frequency comb injection. Second, we analyze the impact of the number of injected comb lines on the time periodic dynamics observed in. ${ }^{17}$ Beside the observation in our previous work as presented in, ${ }^{17}$ we reveal the impact of the number of injected comb lines on the time periodic dynamics which is a new frequency comb region.

Further author information: (Send correspondence to Yaya Doumbia.)

E-mail: yaya.doumbia@centralesupelec.fr, Telephone: +33(0) 387764789 


\section{MATHEMATICAL MODEL}

The laser system is modelled with equations from Ref. ${ }^{1}$ adapted to injection from a comb. The electrical field in the slave laser cavity after injection is defined as: ${ }^{1}$

$$
\frac{d E(t)}{d t}=\left[i \omega(N)+\frac{1}{2}\left(G(N)-\frac{1}{\tau_{p}}\right)\right] E_{S}(t)+E_{M}(t)
$$

Where $E_{S}(t)$ and $E_{M}(t)$ are respectively the complex fields of the slave and the master laser. Here, $\mathrm{i}$ is the imaginary number, $\mathrm{N}$ is the carrier density, $\omega(N)$ and $\mathrm{G}(\mathrm{N})$ are respectively then angular optical frequency and the differential gain of the slave laser and $t_{p}$ is the photon lifetime. The complex electrical field of the injected comb and slave laser can be written as:

$$
\begin{gathered}
E_{M}(t)=\sum_{j} E_{j}(t) e^{i\left(2 \pi \nu_{j} t+\varphi\right)}, \\
E_{S}(t)=E(t) e^{i\left(2 \pi \nu_{0} t+\phi(t)\right.},
\end{gathered}
$$

Where $E_{j}(t)$ and $E(t)$ are the amplitudes of the j-th comb-line and of the slave laser. $\nu_{j}$ and $\nu_{0}$ are the frequencies of the j-th combs-line and of the slave laser. $\Delta \nu_{j}=\nu_{j}-\nu_{0}$ is the detuning between the slave laser and j-th comb mode. $\varphi$ and $\phi$ are respectively the initial phase of each comb-line and the phase of the slave laser. To simplify the calculations, we set the initial phase of each comb-line to 0 and we suppose that the comb modes have the sames amplitudes, $E_{i n j}$. By doing the same calculation as $i^{1}$, the amplitude, phase and the carrier density rate equations can be rewritten as:

$$
\begin{gathered}
\frac{d E}{d(t)}=\frac{1}{2} G_{N}\left(N(t)-N_{t h}\right) E(t)+E_{i n j} \sum_{j} \cos \left(\Phi_{i}(t)-\phi(t)\right), \\
\frac{d \phi}{d(t)}=\frac{1}{2} \alpha G_{N}\left(N(t)-N_{t h}\right)+\frac{E_{i n j}}{E(t)} \sum_{j} \sin \left(\Phi_{i}(t)-\phi(t)\right), \\
\frac{d N}{d(t)}=R_{p}-\frac{N(t)}{\tau_{s}}-G_{N}\left(N(t)-N_{t h}\right) E(t)^{2}-\frac{E(t)^{2}}{\tau_{p}} .
\end{gathered}
$$

In these equations, $N_{t h}$ is the threshold carrier density, $\alpha$ is the linewidth enhancement, $R_{p}$ is the pump rate and ts is the carrier density lifetime. In the following, we shall use $\mathrm{k}$ for the injection strength with $\kappa=\frac{E_{i n j}}{E 0}$ where $E_{0}$ is the field amplitude of the slave laser without optical injections.

Table 1. Semiconductor laser parameters and their numerical.

\begin{tabular}{|l|l|l|}
\hline Differential gain & $G_{N}=7.9 \times 10^{-13}$ & $m^{3} s^{-1}$. \\
\hline Carrier density at threshold & $N_{t h}=2.91924 \times 10^{24}$ & $m^{-3}$. \\
\hline Linewidth enhancement factor & $\alpha=5$ & \\
\hline Pump rate (at threshold) & $R_{p}=1.8 \times 10^{33}$ & $s^{-1}$. \\
\hline Carrier lifetime & $\tau_{s}=2 \times 10^{-9}$ & $s$. \\
\hline Photon lifetime & $\tau_{p}=2 \times 10^{-12}$ & $s$. \\
\hline
\end{tabular}




\section{DIFFERENCE BETWEEN SINGLE MODE INJECTION AND FREQUENCY COMB INJECTION}

\subsection{Injected signal}

The optical injection consists of injecting some light from a laser called master laser in the cavity of a laser called slave laser. On other hand, the laser being a nonlinear oscillator, the optical injection is a way to excite the intrinsic oscillation frequency of the slave laser by an external oscillator. This excitation can be done by a single frequency external oscillator (single mode injection). Figure 1 (a) and (b) show the optical spectrum and the time series respectively for the case of a single mode injection. In this case, the master laser (external oscillator) output is a time stationary signal (Figure $1(\mathrm{~b})$ ), the intrinsic oscillation frequency of the slave laser is excited by only one frequency (Figure 1 (a)). In the case of frequency comb injection, Figure 1 (c) and (d), the injected signal is a non-stationary signal, the slave laser frequency is entrained by several frequencies leading to a modulation of the slave laser output with a frequency of modulation corresponding to the comb spacing $\Omega$. When the detuning between the slave laser frequency and the external oscillator is sufficiently small, the oscillation frequency of the slave laser becomes entrained by the external oscillator leading to so-called optical injection locking.
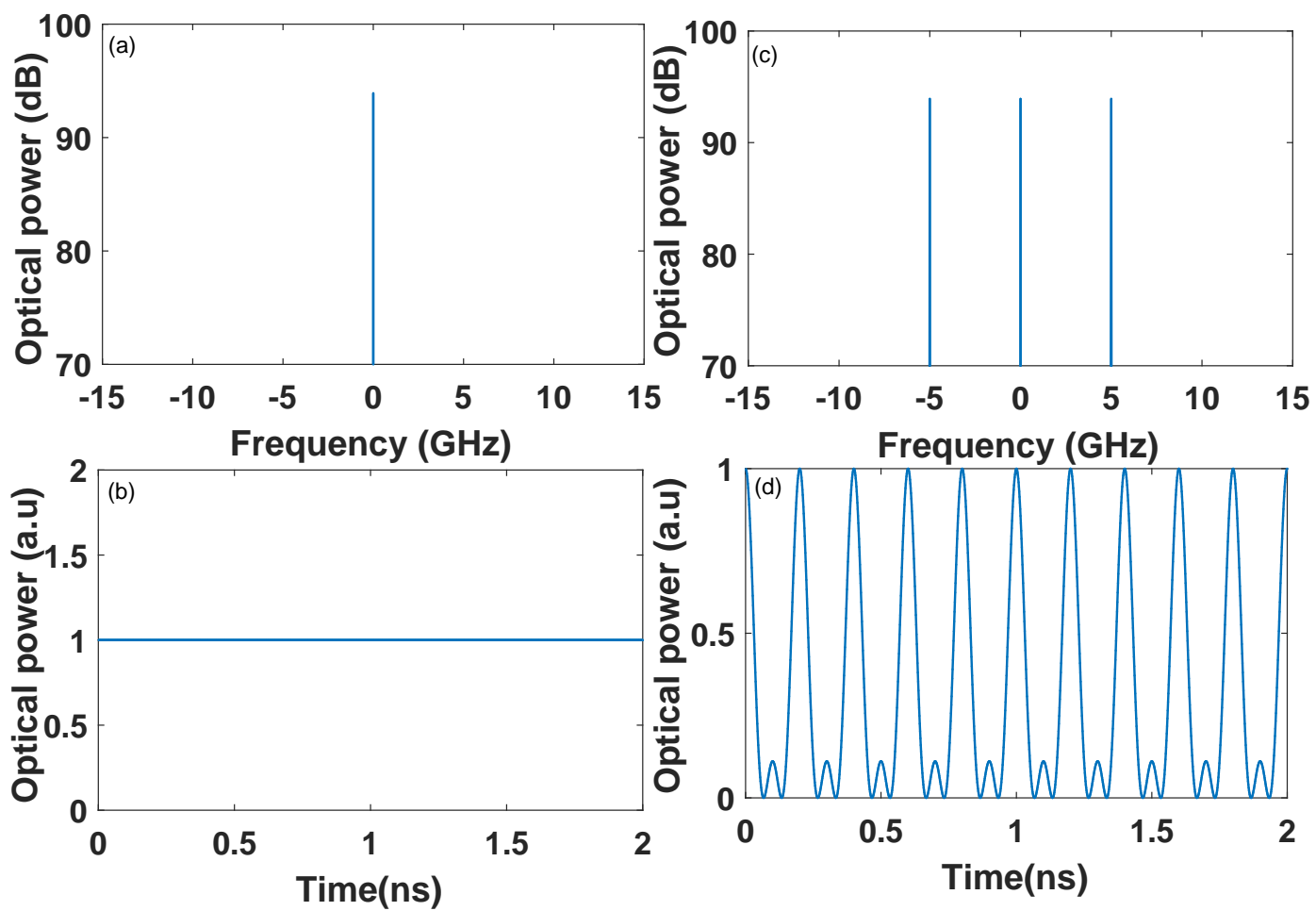

Figure 1. Optical spectra shown in (a) and (c) correspond to single mode and frequency comb master laser output, respectively. Time series in (b) and (d) correspond to single mode and frequency comb master laser output, respectively

\subsection{Optical injection locking}

Figures 2 (a) shows the injection locking solution when the slave laser is subject to single mode optical injection. The red dashed line indicates the free running frequency position of the slave laser. The slave laser being subjected to a single frequency signal, the injection locking solution is characterized by a spectrum with all the power pushed towards the injected signal frequency.

Figure 2 (b) shows the injection locking solution when a master laser is a frequency comb. Unlike to the single frequency injection case, it is not possible to concentrate all the emitted in one frequency. The slave laser 
achieves the injection locking on the comb line that shows the smallest detuning from the slave laser frequency $\Delta \nu$. We define the Comb rejection ratio (CRR) as the difference between the power of the comb line that shows the smallest detuning from the injected comb and the next strongest comb line. We consider injection locking when the CRR is more than $10 \mathrm{~dB}$.
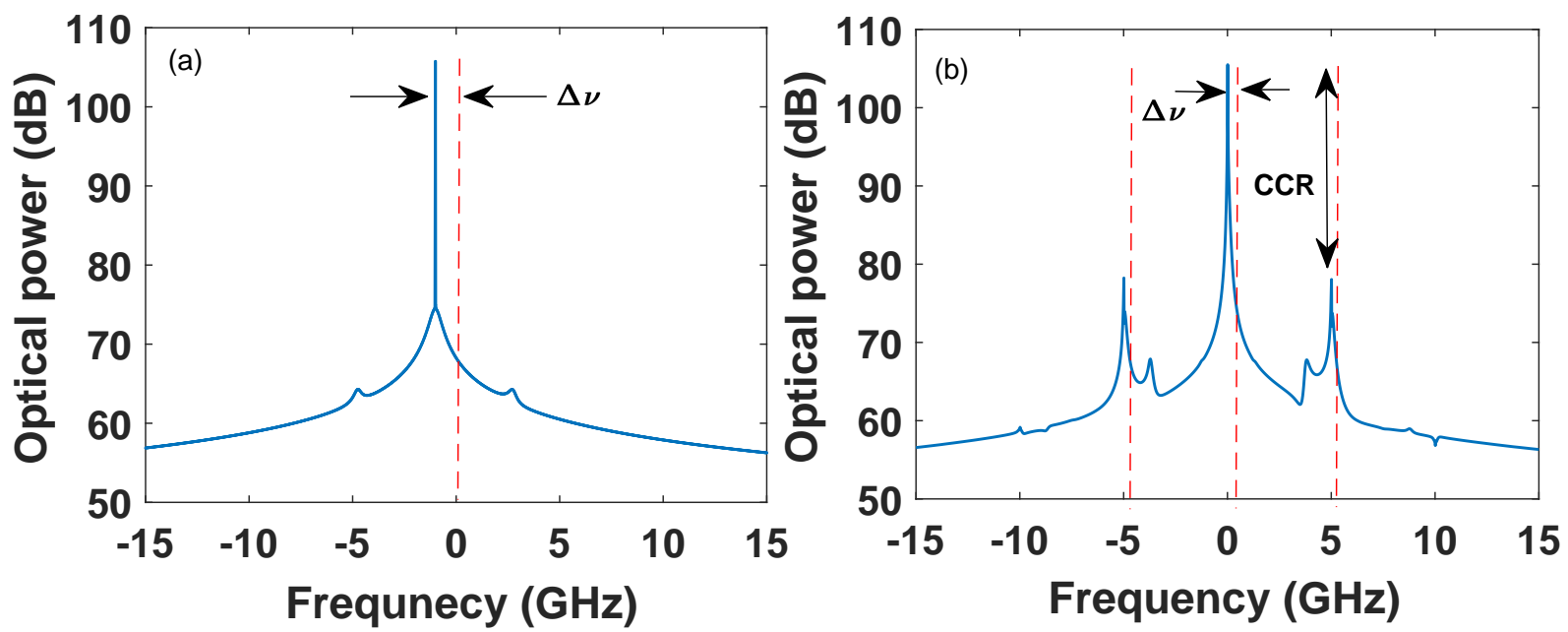

Figure 2. Optical spectra shown in (a) the injection locking solution in the single mode laser (as master laser) injection for a detuning of $\Delta \nu=-1 \mathrm{GHz}$ and injection ratio of $\kappa=0.12$. (b) Injection locking solution in the frequency comb (as master laser) injection case for $\Delta \nu=-0.1 \mathrm{GHz}$ and $\kappa=0.12$.

\section{DYNAMICAL MAPPING}

Figure 3 and 4 show the numerical map in the plan of injection parameter for 9 and 11 comb lines injection, respectively. In the both cases, the comb spacing is $\Omega=5 \mathrm{GHz}$. We have already shown in ${ }^{17}$ that the appearance of the new combs are not due to the comb spacing being close to the relaxation oscillation frequency. To plot these maps, we follow the successive maxima of the time series and compare them to the absolute maximum. When this difference is less than $0-2 \%$ of the maximum amplitude we consider that the dynamics is time-periodic. In these maps, the regions in blue corresponds to the time periodic dynamics which are either the injection locking region called (IL) or the new combs regions called Comb 1 and Comb 2. We have the others dynamics that we consider as chaotic dynamics. As observed in, ${ }^{17}$ we have always two large areas of the new comb region, which extend with the injection injection ratio towards negative detuning. Interestingly, when we increase the number of injected comb line, the regions named comb 1 and comb 2 in $^{17}$ merge and the chaotic dynamics disappear. Additionally, a new comb region is observed to appear around the detuning value of approximately $-10 \mathrm{GHz}$ and becomes more structured when increasing the number of injected comb line.

In figure 5 , we show the optical spectra of unlocked time-periodic dynamics in the regions of comb 1 and comb 2 for 9-comb lines and 11-comb lines injection case. The figures 5 (a) and (b) correspond to the optical spectra for the case of 9 and 11 comb lines injection respectively, in the region of comb 1 for $\kappa=0.8$ and $\Delta \nu=-20.5 \mathrm{GHz}$. For fixed injection parameters and comb, the properties of the new comb i,e, frequency shift, pedestal and shape of optical spectrum remain almost the same. The number of lines in a new comb region has been demonstrated to increase with the number of injected comb line. The figures 5 (c) and (d) show the optical spectra for 9 and 11 comb lines injection for fixed injection ratio to $\kappa=0.8$ and detuning $\Delta \nu=10 \mathrm{GHz}$ respectively in the region of comb 2 . These figures demonstrate that the comb 2 amplifies the number of comb lines generated compared to the comb 1. 


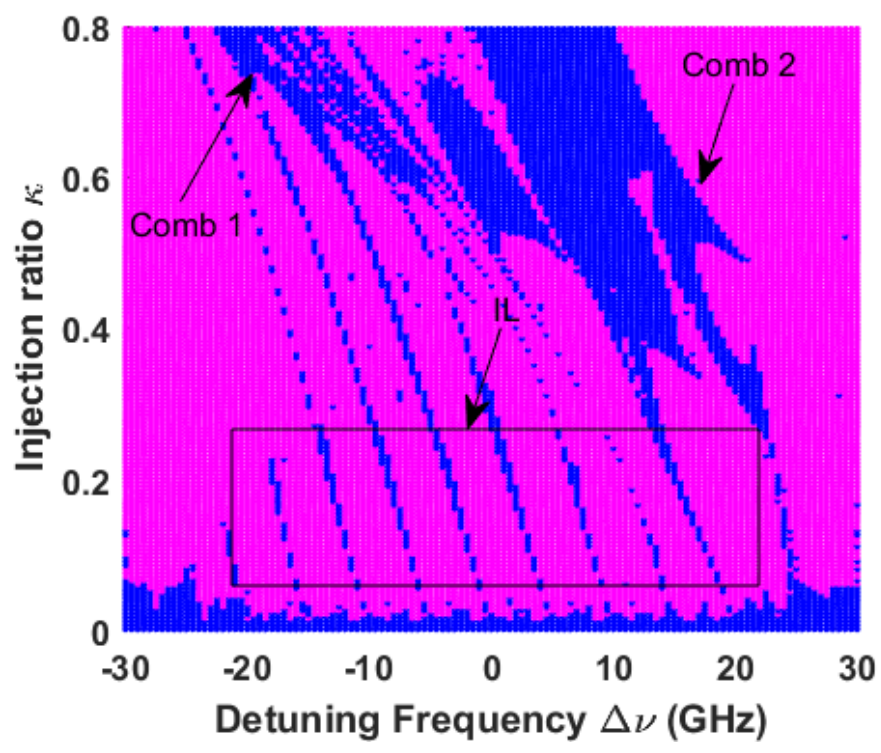

Figure 3. Numerical mapping of semiconductor laser subject to optical injection with frequency comb for 9 comb modes with a comb spacing $\Omega=5 \mathrm{GHz}$. Different regions are observed: IL(Injection locking), Comb1 (First unlocked time periodic dynamics), Comb2(second unlocked time-periodic dynamics) and unlocked chaotic dynamics(magenta part).

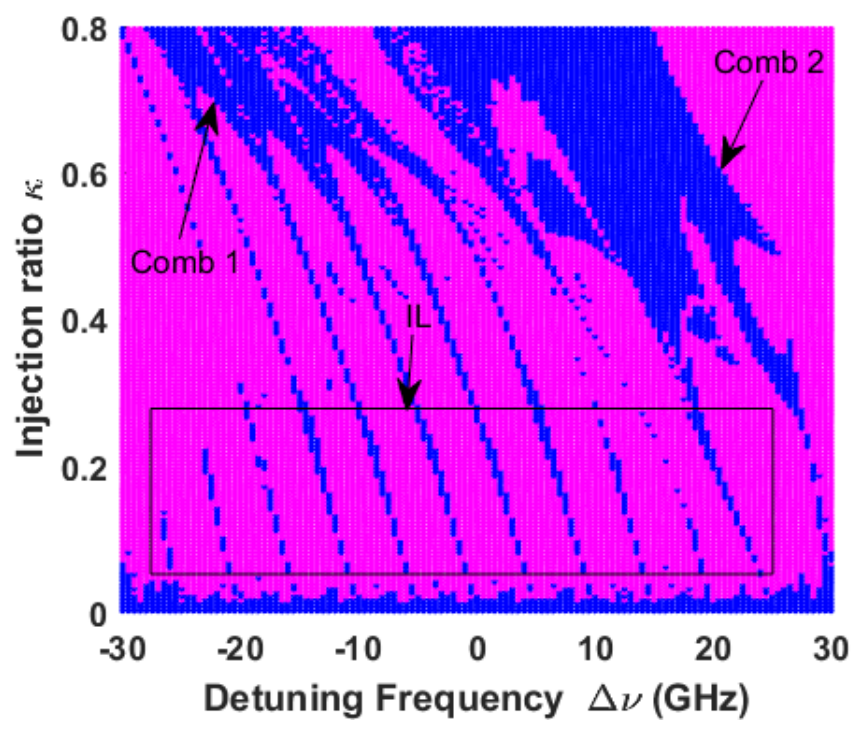

Figure 4. Numerical mapping of semiconductor laser subject to optical injection with frequency comb for 11 comb modes with a comb spacing $\Omega=5 \mathrm{GHz}$. Different regions are observed: IL(Injection locking), Comb1 (First unlocked time periodic dynamics), Comb2(second unlocked time-periodic dynamics) and unlocked chaotic dynamics(magenta part).

\section{CONCLUSION}

In conclusion, we propose the differences between the injection locking solution of a diode laser with single mode injection and frequency comb injection. Unlike to the single mode injection case, with a frequency comb, there is a distribution of the power between the injected comb line when the slave laser is under the injection locking condition. A broadened optical frequency comb (new comb) is also observed when we inject a frequency comb. 

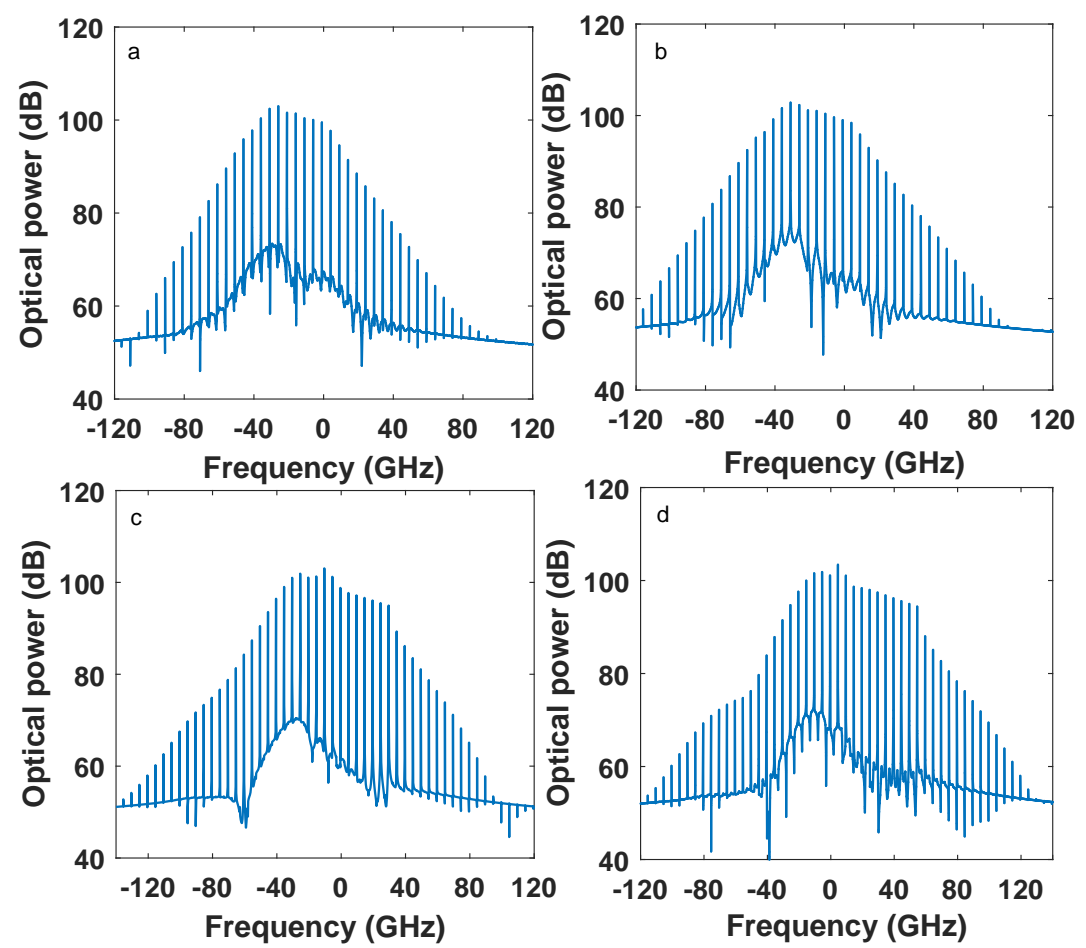

Figure 5. Optical spectra when varying the detuning and the number of injected comb line for fixed injection ratio $\kappa=0.8$. (a) and (b) correspond to a detuning of $\Delta \nu=-20.5 \mathrm{GHz}$ for 9 and 11-comb lines injection. (c) and (d) correspond to a detuning of $\Delta \nu=10 \mathrm{GHz}$ for 9 and 11-comb lines injection

The new combs regions extend and change with the number of injected comb lines and other injection parameters. The new comb regions become more structured in the plan of the injection parameters when we increase the number of injected comb lines.

Funding : Ministère de l'Enseignement supérieur, de la Recherche et de l'Innovation; Région Grand-Est; Département Moselle; European Regional Development Fund (ERDF); Metz Métropole; Airbus GDI Simulation; CentraleSupélec; Fondation CentraleSupélec; Fonds Wetenschappelijk Onderzoek (G0E5819N).

\section{REFERENCES}

[1] Mogensen, F., Olesen, H., and Jacobsen, G., "Locking conditions and stability properties for a semiconductor laser with external light injection," IEEE Journal of Quantum Electronics 21(7), 784-793 (1985).

[2] Gatare, I., Sciamanna, M., Nizette, M., and Panajotov, K., "Bifurcation to polarization switching and locking in vertical-cavity surface-emitting lasers with optical injection," Physical Review A 76(3), 031803 (2007).

[3] Simpson, T., "Mapping the nonlinear dynamics of a distributed feedback semiconductor laser subject to external optical injection," Optics Communications 215(1-3), 135-151 (2003).

[4] Sciamanna, M. and Shore, K. A., "Physics and applications of laser diode chaos," Nature photonics 9(3), 151 (2015).

[5] Sivaprakasam, S. and Shore, K., "Signal masking for chaotic optical communication using external-cavity diode lasers," Optics letters 24(17), 1200-1202 (1999).

[6] Lin, L.-C., Liu, S.-H., and Lin, F.-Y., "Stability of period-one (p1) oscillations generated by semiconductor lasers subject to optical injection or optical feedback," Optics express 25(21), 25523-25532 (2017). 
[7] Dong, B., Huang, H., Duan, J., Kurczveil, G., Liang, D., Beausoleil, R. G., and Grillot, F., "Frequency comb dynamics of a $1.3 \mu \mathrm{m}$ hybrid-silicon quantum dot semiconductor laser with optical injection," Optics letters 44(23), 5755-5758 (2019).

[8] Gatare, I., Sciamanna, M., Buesa, J., Thienpont, H., and Panajotov, K., "Nonlinear dynamics accompanying polarization switching in vertical-cavity surface-emitting lasers with orthogonal optical injection," Applied Physics Letters 88(10), 101106 (2006).

[9] Valle, A., Gatare, I., Panajotov, K., and Sciamanna, M., "Transverse mode switching and locking in verticalcavity surface-emitting lasers subject to orthogonal optical injection," IEEE Journal of Quantum Electronics 43(4), 322-333 (2007).

[10] Denis-le Coarer, F., Quirce, A., Valle, Á., Pesquera, L., Sciamanna, M., Thienpont, H., and Panajotov, K., "Polarization dynamics induced by parallel optical injection in a single-mode vcsel," Optics letters 42(11), 2130-2133 (2017).

[11] Olejniczak, L., Panajotov, K., Thienpont, H., and Sciamanna, M., "Self-pulsations and excitability in optically injected quantum-dot lasers: Impact of the excited states and spontaneous emission noise," Physical Review A 82(2), 023807 (2010).

[12] Wang, C., Raghunathan, R., Schires, K., Chan, S.-C., Lester, L. F., and Grillot, F., "Optically injected inas/gaas quantum dot laser for tunable photonic microwave generation," Optics letters 41(6), 1153-1156 (2016).

[13] Duill, S. P. Ó., Anandarajah, P. M., Smyth, F., and Barry, L. P., "Injection-locking criteria for simultaneously locking single-mode lasers to optical frequency combs from gain-switched lasers," in [Physics and Simulation of Optoelectronic Devices XXV], 10098, 100980H, International Society for Optics and Photonics (2017).

[14] Wu, D. S., Slavík, R., Marra, G., and Richardson, D. J., "Phase noise and jitter characterization of pulses generated by optical injection locking to an optical frequency comb," in [Frontiers in Optics], FW2A-3, Optical Society of America (2012).

[15] Wu, D. S., Richardson, D. J., and Slavík, R., "Selective amplification of frequency comb modes via optical injection locking of a semiconductor laser: influence of adjacent unlocked comb modes," in [Integrated Optics: Physics and Simulations], 8781, 87810J, International Society for Optics and Photonics (2013).

[16] Shortiss, K. J., Shayesteh, M., and Peters, F. H., "Modelling the effect of slave laser gain and frequency comb spacing on the selective amplification of injection locked semiconductor lasers," Optical and Quantum Electronics 50(1), 49 (2018).

[17] Doumbia, Y., Malica, T., Wolfersberger, D., Panajotov, K., and Sciamanna, M., "Optical injection dynamics of frequency combs," Optics Letters 45(2), 435-438 (2020). 